\title{
Lidil
}

Revue de linguistique et de didactique des langues

$40 \mid 2009$

La motivation pour l'apprentissage d'une langue seconde

\section{Un ménage à trois fragile : Autonomie, Motivation et Apprentissage dans un Centre de Langues}

\section{Peter Prince}

\section{CpenEdition}

\section{Journals}

Édition électronique

URL : http://journals.openedition.org/lidil/2925

DOI : $10.4000 /$ lidil.2925

ISSN : 1960-6052

Éditeur

UGA Éditions/Université Grenoble Alpes

Édition imprimée

Date de publication : 1 décembre 2009

Pagination : $71-88$

ISBN : $978-2-84310-154-0$

ISSN : $1146-6480$

Référence électronique

Peter Prince, « Un ménage à trois fragile : Autonomie, Motivation et Apprentissage dans un Centre de Langues », Lidil [En ligne], 40 | 2009, mis en ligne le 01 juin 2011, consulté le 10 décembre 2020. URL http://journals.openedition.org/lidil/2925 ; DOI : https://doi.org/10.4000/lidil.2925 


\title{
Un ménage à trois fragile : Autonomie, Motivation et Apprentissage dans un Centre de Langues
}

\author{
Peter Prince*
}

\begin{abstract}
RÉSUMÉ
En matière d'apprentissage, autonomie et motivation sont étroitement liées. Un apprenant pleinement autonome est en général motivé, mais ce n'est pas toujours le cas que les apprenants veulent être autonomes. La question de l'autonomie se pose avec acuité dans un Centre d'Apprentissage des Langues, où il s'agit de maintenir la motivation des apprenants tout en les amenant sur le chemin de l'autonomie. Après une discussion des fondements théoriques du rapport entre les deux concepts, nous présentons les résultats d'une enquête qui met en évidence, d'une part, le caractère multiforme de l'autonomie, d'autre part, l'importance de la confiance chez les apprenants.
\end{abstract}

\section{ABSTRACT}

When it comes to learning, autonomy and motivation are closely linked. A fully autonomous learner is generally motivated, but not all learners want to be autonomous. The question of autonomy is crucial to a Language Centre, where learners' motivation needs to be maintained as they are guided on the path to autonomy. After discussing the theoretical basis of the link between the two concepts, we present the results of a study which highlights, on the one hand, the multiple forms autonomy can take, and on the other the importance of confidence in the learners.

Il y a trois ans, suite à un voyage au Japon, un ami m'a fait part de son intention d'apprendre le japonais. Il habite un village où personne ne peut lui donner de cours; il avait donc comme seul matériel des cédéroms et des livres. Une initiative qui n'a, sans doute, pas fait long feu?

* Université de Provence. 
Pas du tout : contrairement à ce que l'on pourrait supposer, il continue toujours.

Un tel exemple, même s'il est exceptionnel, démontre qu'il est possible d'apprendre seul une langue étrangère. La première condition est de maintenir sa motivation; la deuxième est de faire preuve d'autonomie, c'est-à-dire s'astreindre à un travail régulier et trouver dans les progrès accomplis, aussi lents soient-ils, la motivation nécessaire pour continuer.

La promotion de l'autonomie fait souvent partie des objectifs explicites affichés dans les Centres d'apprentissage des langues (CAL). Nous nous proposons ici d'examiner en quoi consiste l'autonomie, avant de présenter quelques données issues d'une recherche-action menée au CAL de l'Université de Provence. L'objectif de celle-ci était double : d'une part tester l'apport d'un questionnaire dans le dispositif du centre; d'autre part examiner l'évolution de l'autonomie au cours d'une formation au CAL.

\section{Qu'est-ce que l'autonomie?}

Benson (2001) définit l'autonomie comme la «capacité à prendre le contrôle de son apprentissage ». Il s'ensuit que l'autonomie varie selon les personnes, car chaque individu possède cette capacité à un degré différent. L'autonomie commence par le sentiment général de pouvoir accomplir par soi-même ce que l'on entreprend, sans être déstabilisé par une situation nouvelle. Pour un apprenant confiant, l'espace d'apprentissage que l'on trouve dans un CAL est perçu comme une occasion à saisir, un endroit où cette capacité peut trouver toute son expression. Est-ce à dire que seuls les CAL permettent un tel épanouissement? Si pour Benson (2001, p. 114), ils occupent en effet une place centrale dans la pratique de l'autonomie, il serait trompeur de les opposer au présentiel comme s'il s'agissait de deux modes d'apprentissage distincts. Placer la notion de contrôle au cœur de l'autonomie implique plutôt que l'on s'interroge sur le degré de contrôle exercé, et par qui, dans un environnement d'apprentissage donné.

Sur le plan cognitif, en matière de contrôle, le lieu où il se situe est déterminant. Le lieu de contrôle peut être externe à l'apprenant, lorsque quelqu'un d'autre détermine ce qui est à faire, ou interne, lorsque les choix sont effectués par l'apprenant. Benson propose une classification en trois niveaux : contrôle de l'administration de la formation, du 
contenu de la formation et des processus cognitifs et métacognitifs mis en œuvre.

Le transfert éventuel d'une partie au moins des deux premiers types de contrôle vers l'apprenant découle de l'organisation administrative et matérielle d'une formation qui a lieu dans un CAL. Ce ne sont là, pourtant, que des conditions de départ; exercer le contrôle à bon escient, en choisissant les ressources pédagogiques les plus pertinentes, suppose que l'apprenant ait une idée claire, d'une part, de ses objectifs, d'autre part de la manière dont les ressources en question peuvent l'en rapprocher. En d'autres termes, l'autonomie sur le plan matériel ne portera ses fruits que si l'apprenant est capable aussi d'exercer le troisième type de contrôle, sur les processus cognitifs et métacognitifs.

Apprendre suppose une allocation de ressources attentionnelles, non seulement pour accomplir une tâche, mais aussi pour assurer le contrôle du niveau cognitif : recherche de liens avec des connaissances stockées en mémoire, travail de mémorisation, formulation d'hypothèses nouvelles. Ce travail implique une double prise de conscience de la part de l'apprenant: d'une part que la langue cible fonctionne comme un système propre, avec des similitudes et des différences par rapport à sa L1 ; d'autre part que l'apprentissage passe par le renforcement et l'enrichissement de représentations lexicales et syntaxiques. Cette prise de conscience amène l'apprenant vers le niveau métacognitif, où il réfléchit à la fois au matériel à apprendre et à la manière de l'apprendre. L'exercice d'un contrôle cohérent de ce niveau est sans doute la meilleure indication d'une autonomie pleinement assumée, et le meilleur garant d'un apprentissage réussi au CAL. La connaissance métacognitive est classée par Flavell (1979) en trois catégories : la connaissance de soi-même (ses capacités, sa motivation), de la tâche à effectuer (ses composantes, sa finalité) et des stratégies utilisables. Pour Wenden (1998), cette connaissance métacognitive est le fondement de l'auto-régulation, qui permet à l'apprenant de prendre le recul nécessaire pour planifier une activité, surveiller la manière dont elle se déroule, et évaluer à la fois sa propre performance et l'utilité de l'activité à la fin. L'auto-régulation est favorisée lorsque l'apprenant a une motivation intrinsèque, un sentiment d'auto-efficacité et une perception positive de la valeur de la tâche qu'il entreprend (Pintrich, 1999).

$\mathrm{Ne}$ serait-ce que par son agencement physique et l'absence du face à face avec un enseignant, le CAL offre d'emblée l'occasion d'entamer une réflexion métacognitive. Laisser à l'apprenant le choix des activités l'incite en effet à se poser des questions sur la pertinence ou la difficulté 
de celles-ci, ainsi que sur sa propre motivation à les faire. C'est déjà le début d'une prise en charge de son apprentissage, et de nombreux apprenants épousent avec plaisir et efficacité l'autonomie qui leur est proposée. Ceux-là accordent une importance à l'apprentissage d'une langue, se sentent en mesure de progresser grâce aux moyens dont ils disposent, et sont habitués à réfléchir en termes d'objectifs. Ils planifient leurs activités en fonction du nombre et de la durée des séances au CAL, de la connaissance qu'ils ont des ressources disponibles et de l'objectif qu'ils visent. Les représentations qu'ils ont du CAL forment ainsi un réseau cohérent, auquel participent des connaissances métacognitives qu'ils activent chaque fois que nécessaire afin de réguler leur parcours. Ces connaissances fonctionnent comme des signaux, permettant de déclencher, guider et maintenir l'activité cognitive. Le contrôle de l'attention chez l'apprenant pleinement autonome est peu couteux sur le plan cognitif car rien ne vient la détourner.

Force est de constater que tous ne font pas preuve d'une telle autonomie. D'abord, parce que la réflexion métacognitive, bien que très utile, n'est pas indispensable à l'apprentissage d'une langue. Un apprenant dont les enseignants ont contrôlé toutes les dimensions du parcours aura du mal à se constituer une base de connaissances métacognitives. Deuxièmement, l'autonomie implique une confiance en soi (Bandura, 2003) qui permet à l'apprenant d'expérimenter car son expérience passée lui a montré qu'il est capable d'apprendre non seulement de ses réussites mais, plus important, de ses échecs. Enfin, s'interroger sur la capacité d'un apprenant d'exercer un contrôle sur son apprentissage nous amène à prendre en compte sa motivation. En effet, on peut être motivé pour apprendre une langue sans être motivé pour le faire de manière autonome. La définition de la motivation proposée par Raby (2007) pour tenir compte d'un contexte d'apprentissage autonome confirme cette idée. À côté des trois composantes habituellement citées (désir et effort appliqués au but d'apprendre une langue) elle ajoute le désir de prendre des initiatives et de réguler son activité d'apprentissage. Ces deux derniers aspects sont dissociables des autres et rien ne garantit qu'ils en découleront automatiquement. Pour de nombreux chercheurs, pourtant, autonomie et motivation entretiennent des relations étroites. Le postulat de base concernant cette relation est avancé par Deci et Ryan $(1985,2002)$ : la motivation à entreprendre ou à poursuivre une action donnée augmente dès lors que l'on s'estime en mesure de contrôler le processus en jeu. 
On retrouve la notion de contrôle : s'agissant du processus d'apprentissage, Deci et Ryan placent la perception du lieu de contrôle (locus of control) au centre de leur théorie. Une vraie autonomie suppose que l'individu choisisse une action en toute liberté - il aurait pu aussi bien choisir de ne pas la faire. Son comportement est alors auto-déterminé (self-determined), et sa motivation intrinsèque - l'effort étant librement consenti, il trouve une satisfaction dans l'accomplissement de la tâche et non pas dans une récompense extérieure. De ce fait, selon Ushioda (1996), une motivation qui est instrinsèque est étayée par elle-même.

L'argument pédagogique en faveur des CAL semble donc bien établi, puisque les CAL favorisent l'autonomie et l'autonomie favorise la motivation. La réalité, pourtant, n'est pas aussi simple, car comme le souligne Albero (2002), elle résulte de choix plus ou moins bien réfléchis, ou parfois même paradoxaux, dont, entre autres, le «choix de l'individualisation pour gérer la massification et celui des technologies pour pallier des manques de moyens ». Le personnel du CAL devra ainsi naviguer entre deux écueils : d'une part une institution tentée de ne voir dans le CAL qu'une manière peu onéreuse d'offrir une formation en langues à des masses d'étudiants ; d'autre part des apprenants prompts à réduire le CAL à un travail individuel devant l'ordinateur, ce qui n'est pas en soi une expression d'autonomie. Albero note par ailleurs que chaque dispositif d'autoformation est porteur d'une histoire et d'un projet qui lui sont propres, et que son potentiel autonomisant ou motivant dépend du contexte dans lequel il évolue. Sur le terrain, donc, la théorie de l'auto-détermination se heurte à des réalités diverses, où l'on voit qu'il ne suffit pas de donner à l'apprenant l'occasion de devenir autonome pour que sa motivation augmente, ou change de nature pour devenir plus intrinsèque, et ce n'est pas parce que l'environnement d'apprentissage devient non contrôlant que tous les apprenants découvriront l'autonomie. La théorie veut qu'un apprenant autonome soit motivé, et le CAL lui offre la possibilité d'une grande autonomie, mais il s'avère souvent qu'il n'est pas en mesure de s'approprier l'autonomie proposée. Du côté de l'apprenant, cette difficulté peut être imputée à un manque d'habitude, de confiance ou de motivation, ou bien à une combinaison de ces trois facteurs. Du côté du CAL, réduire la difficulté suppose que soient mises en œuvre les trois conditions nécessaires à la réussite de l'apprentissage auto-dirigé : la formation des apprenants sur le plan méthodologique, la mise à disposition de ressources adéquates, et la formation de tuteurs ou conseillers (Holec, 2000). 
Au risque de simplifier, nous résumons la discussion jusqu'ici en suggérant que la capacité à être autonome dépend de trois facteurs principaux : une confiance en soi en matière d'apprentissage, une base solide de connaissances métacognitives et une motivation intrinsèque. Lorsque ces trois facteurs sont réunis, l'apprenant est déjà autonome, et pourra profiter pleinement du CAL. L'absence de ces facteurs ne signifie nullement que l'autonomie n'est pas possible, car le propre d'une capacité est d'exister aussi sous forme potentielle. Son développement sera cependant plus problématique, accompagné parfois de désarroi, voire de résistances. Face à ces différents cas de figure, il importe pour le CAL de pouvoir réagir rapidement de manière appropriée. Cela implique que l'on puisse dépister des difficultés éventuelles dès le début de la formation, ce qui à son tour, implique que l'on dispose d'un instrument de mesure approprié.

Il est clair que l'autonomie ne se prête pas à une mesure directe. Il n'existe d'ailleurs aucune échelle reconnue qui permettrait même une mesure indirecte. Sinclair (1999) offre une piste intéressante avec une classification des énoncés des apprenants en fonction du niveau de réflexion métacognitive qu'ils démontrent. Il s'agit toutefois d'un continuum plutôt que de catégories distinctes, et la classification repose sur des indices langagiers très variables, donc difficiles à formaliser.

Une autre approche, issue de l'ergonomie cognitive, passe par une observation fine de l'interaction entre apprenants et dispositif d'apprentissage (Raby, Baillé, Bressoux et Chapelle, 2003). Ce qui ressort, entre autres, de cette démarche féconde est la difficulté à conceptualiser l'autonomie autrement qu'en situation : tel individu est plus ou moins autonome en fonction de la tâche à effectuer et de ses propres connaissances et représentations.

Pour sa part, Cotterall $(1995,1999)$ a utilisé des questionnaires pour sonder la disposition à l'autonomie des apprenants (readiness for autonomy). Elle note la méfiance qu'il convient d'adopter par rapport à ces analyses quantitatives, dont les facteurs mis en évidence sont instables, tout en affirmant l'intérêt de cette approche :

If an appropriate methodology can be found, future research could explore whether "profiles" of learner beliefs can be identified. The goal of such research is not to assign learners to rigid categories, but rather to further our understanding of the framework within which language learners operate. (Cotterall, 1999, p. 510) 
Ces différentes manières d'aborder l'autonomie montrent non seulement à quel point elle est fluide, prenant des formes différentes selon les circonstances, mais aussi la nécessité, pour celui qui se penche sur la question, d'être lucide sur les objectifs et limites de sa démarche. Un questionnaire peut viser à dégager des constantes généralisables, mais celles-ci seront alors grossières car dissociées d'un contexte donné. Il sera alors souhaitable de vérifier les profils dégagés en observant les comportements au CAL et en examinant ce qui en résulte en tant que productions (Raby, 2007). Le questionnaire, en revanche, a l'avantage d'être rapide et facile à administrer.

\section{Une étude quantitative au CAL de I'Université de Provence}

La recherche-action rapportée ici avait pour principal objectif de tester l'apport éventuel d'un questionnaire (voir annexe 1) dans le dispositif d'ensemble du CAL. Dans l'optique adoptée, le questionnaire et le tutorat forment un tout, le premier permettant au tuteur de savoir où se situe l'apprenant et ainsi de mieux faciliter l'apprentissage ${ }^{1}$. On peut dire ainsi qu'un premier objectif du questionnaire, dans la lignée de Cotterall (1999), est de cerner la disposition des apprenants par rapport à l'autonomie au tout début de la formation.

Le questionnaire énumère certaines actions rentrant dans l'apprentissage d'une langue. Pour chaque action l'apprenant estime s'il a besoin ou non d'un professeur, donnant lieu à un score entre 0 (tout à fait besoin) et 5 (pas du tout besoin). Plus le score est élevé, plus on peut considérer que l'apprenant est disposé à l'autonomie. Dans le cas de cette étude, il était clairement signalé que se dispenser d'un professeur ne signifie pas forcément travailler seul, mais offre la possibilité de travailler avec d'autres étudiants (pour une discussion approfondie des différentes modalités de travail en groupe, voir Mangenot et Nissen, 2006).

Le choix précis des items résulte d'une réflexion menée entre plusieurs enseignants sur l'acte même d'enseigner. Que fait un enseignant de langues pour favoriser l'apprentissage ? Répondre à cette question

1. Si le tutorat n'est pas abordé ici, c'est qu'il mérite une discussion plus ample. Voir par exemple Narcy-Combes (2003), Mangenot et Nissen (2006), Mozzon-McPherson (2007). 
revient à définir les composantes les plus importantes de son rôle dans la salle de classe. Ensuite il s'agit de savoir si, dans un CAL, ces composantes peuvent être transférées aux apprenants travaillant de manière individuelle ou en groupe.

L'analyse factorielle des réponses au questionnaire a donné lieu à trois ensembles de corrélations. Celles-ci concernent 10 des 18 items traitant de l'autonomie, les autres ne rentrant pas dans des corrélations de manière stable à travers différents groupes d'apprenants. Un facteur appelé «contrôle du processus » (items $7,10,11,18$ ) touche au besoin d'un professeur pour choisir le rythme du travail, définir les objectifs, stimuler à travailler et diriger les activités. À priori, pour un apprenant dont le score est bas sur ces items, la perspective de devoir prendre le contrôle des aspects pratiques de son apprentissage ne l'enchante guère; il doute de sa capacité à y arriver. Cet aspect de l'autonomie est pourtant celui auquel il est confronté le plus tôt dans un CAL, où dès la première séance il doit assumer certaines responsabilités laissées autrefois à l'enseignant.

Le facteur «recherche de feedback» (items 2 et 13) traduit le besoin d'un professeur pour évaluer le travail et corriger les erreurs. D'après les résultats de Cotterall (1999), c'est la capacité la moins développée chez les apprenants. Elle trouve cela surprenant, car «editing and other monitoring activities are common in most language classes» (p. 509). Bien entendu, le terme «évaluation» recouvre plusieurs pratiques, de l'attribution d'une note au feedback formatif. Si l'on considère que, au-delà des différentes formes qu'elle peut prendre, ce que l'élève retient surtout est l'expertise de l'enseignant en la matière, le constat de Cotterall est moins surprenant. La pratique de l'auto-évaluation suppose ainsi que le CAL mette à disposition un matériel adéquat et que l'apprenant adhère à la démarche.

Enfin, un troisième groupe de 4 items $(1,5,15,16)$ constituant un facteur «recherche d'explications», concerne le besoin d'un professeur pour expliquer le sens d'un texte, les points de grammaire, le vocabulaire utile et les références culturelles. Un score élevé ici implique que l'apprenant se sent capable, non seulement de décider si oui ou non tel élément mérite un éclaircissement, mais aussi de procéder à cet éclaircissement lui-même.

À côté des items traitant de l'autonomie, le questionnaire comporte une série de 10 items qui montrent une forte corrélation entre eux, et qui reflète un facteur «confiance». Clément, Dörnyei et Noels (1994) sou- 
lignent l'importance de ce facteur : douter de sa capacité à faire quelque chose peut fortement affecter sa motivation à le faire.

En tant qu'outil, le questionnaire constitue ainsi un moyen rapide de situer un apprenant par rapport à l'autonomie dès le début de sa formation. En fonction des résultats, il est possible de planifier une séance de tutorat rapidement pour ceux qui montrent un profil peu autonome et/ou peu confiant. Disposer de cette information avant de rencontrer l'apprenant signifie que le tuteur tâtonne moins et peut plus facilement engager un dialogue constructif avec l'apprenant. L'apport du questionnaire a donc été jugé positif, malgré son caractère approximatif.

Le deuxième objectif de la recherche-action consistait à cerner l'évolution de l'attitude des apprenants suite à leur formation au CAL. En effet, administré en fin de formation, le questionnaire devient un outil permettant d'étudier l'évolution de la disposition à l'autonomie suite à l'expérience pratique du CAL, et d'ajuster le dispositif en fonction des tendances qui se dégagent. Les données que nous présentons ici concernent 44 étudiants en troisième année de licence pluridisciplinaire qui ont rempli le questionnaire au début et en fin de formation.

Le score moyen sur 5 a été calculé pour les deux dimensions, autonomie et confiance. Pour l'autonomie un score de 0 a été attribué à chaque réponse +++ (je suis tout à fait d'accord) et 5 à une réponse --- (je ne suis pas du tout d'accord). Le même système a été utilisé pour la confiance, sauf pour les items $21,23,31$ et 34 , où les scores étaient inversés. La progression entre le début et la fin de la formation est significative en ce qui concerne la confiance (2,46 au début contre 2,69 à la fin, $\mathrm{F}(1,43)=14,27$, p <.0005) mais la progression en autonomie est moindre $(2,51$ au début contre 2,63 à la fin, $F(1,43)=1,95$, n.s.). Le score moyen pour l'autonomie cache toutefois des différences entre les trois facteurs identifiés, visibles dans le tableau 1.

\begin{tabular}{|l|c|c|}
\cline { 2 - 3 } \multicolumn{1}{c|}{} & pré & post \\
\hline Contrôle du processus & 3,3 & 3,4 \\
\hline Recherche de feedback & 1,2 & 1,5 \\
\hline Recherche d'explications & 1,3 & 1,8 \\
\hline
\end{tabular}

Tableau 1. - L'évolution de l'autonomie au cours d'une formation au CAL (scores moyens sur 5). 
On constate que l'autonomie ne s'exerce pas de manière uniforme. Le contrôle du processus dans ses aspects pratiques semble plus accessible que la prise en charge du feedback ou des explications. Malgré une légère progression, les scores pour ces deux facteurs restent plutôt bas, même en fin de formation. Cette différence s'explique par le fait que, pour contrôler le processus, il suffit d'étendre une capacité déjà largement utilisée dans la vie quotidienne, alors que les deux autres facteurs font appel à des capacités métacognitives jusque-là inexplorées, car supposées être l'apanage de l'enseignant.

Aussi juste que cette interprétation puisse être, la nature du projet du CAL rend difficile d'en rester là. Ne peut-on ou ne doit-on élaborer des outils permettant aux apprenants de développer une autonomie dans ces domaines aussi? La réponse dépendra de l'importance que l'on donne à l'autonomie comme objectif en tant que tel. S'il est légitime de penser que ce qui compte avant tout est le progrès linguistique, cela n'exclut pas de promouvoir l'autonomie autant que possible. Des entretiens avec les apprenants, menés également en fin de formation, font apparaitre que pouvoir exercer un contrôle sur les aspects pratiques du processus est motivant (des extraits sont donnés en annexe 2, suivis d'un bref commentaire; voir aussi Prince, 2008). Si on peut les amener à une autonomie dans les deux autres facteurs, ne verrait-on pas des conséquences positives semblables? Si l'on en croit Ushioda (1996), pour qui «Autonomous language learners are by definition motivated learners » (p. 2), ce sera forcément le cas. Cela suppose néanmoins deux conditions : que l'apprenant veut le faire et qu'il pense qu'il peut le faire. Si les apprenants sont en général ouverts à un mode d'apprentissage en lequel ils fondent un certain espoir, ils ne sont pas forcément prêts à pousser leur effort vers la réflexion métacognitive qu'une réelle autonomie implique.

Un autre renseignement notable qui ressort de cette étude concerne le rapport entre autonomie et confiance. Le tableau 2 présente l'évolution de l'autonomie pour les trois facteurs en fonction du niveau de confiance exprimé au début de la formation. Ces données ont été obtenues en regardant, sur les 44 participants, les réponses des 15 plus confiants et des 15 moins confiants. Ensuite l'évolution des attitudes a été examinée en comparant les réponses des deux groupes au début et à la fin (comparaison - pré / - post pour les moins confiants, + pré / + post pour les plus confiants). On constate une corrélation générale, où les plus confiants sont les plus autonomes, au début comme à la fin de la formation. 


\begin{tabular}{|l|c|c|c|c|}
\cline { 2 - 5 } \multicolumn{1}{c|}{} & - pré & - post & + pré & + post \\
\hline Contrôle du processus & 2,4 & 3,0 & 4,1 & 3,9 \\
\hline Recherche de feedback & 0,9 & 1,1 & 1,6 & 1,8 \\
\hline Recherche d'explications & 1,3 & 1,2 & 1,6 & 2,4 \\
\hline
\end{tabular}

Tableau 2. - L'évolution de l'autonomie selon le degré de confiance des apprenants (scores moyens sur 5 ).

La différence se fait le plus ressentir pour la recherche d'explications, où les moins confiants enregistrent une légère baisse en fin de formation et les plus confiants une hausse sensible. On peut voir dans ce résultat la confirmation que l'exercice de l'autonomie a des conséquences positives - sinon les plus confiants ne l'auraient pas exercée. Plus important, on constate que la confiance est primordiale. Pour prendre l'exemple de la grammaire : les outils à disposition sont les mêmes pour tous, mais seuls les plus confiants ont mis à profit une capacité à s'en servir. Le problème n'est pas tant du côté de l'outil - les manuels de grammaire clairs et accessibles ne manquent pas - que de celui de la perception de sa propre capacité par l'apprenant. Moins celui-ci est confiant, moins il se sent capable de sérier les difficultés : la grammaire reste un tout, aussi flou qu'insaisissable, source de nombreuses difficultés passées, en apparence encore insurmontables. Le manuel a beau être clair aux yeux d'un expert, pour lui il reste opaque. La conséquence, dans ce jeu subtil entre vouloir et pouvoir, est que dès qu'il sent qu'il ne peut pas, sa motivation est trop fragile pour qu'il continue à vouloir.

\section{Conclusion}

Bien que complétée par des entretiens qui ont fourni des informations supplémentaires, cette étude s'inscrit dans une démarche essentiellement quantitative. Les limites de questionnaires comme moyen de cerner une réalité complexe sont bien connus : ce qui est décrit ici ne couvre donc pas l'ensemble de cette réalité. Il manque notamment l'observation détaillée des apprenants en situation, qui permettrait de vérifier comment les représentations révélées par le questionnaire se traduisent en actes. Une telle vérification, par le biais d'une étude triangulaire telle que préconisée par Raby (2007), serait nécessaire pour mieux comprendre l'interaction spécifique entre un apprenant et un 
support pédagogique donné, ce qui ouvre la voie à des moyens d'actions pour accroitre l'efficacité de celle-ci.

Malgré son caractère forcément incomplet, cette étude permet néanmoins d'examiner de plus près le rapport entre autonomie et motivation. On voit ainsi confirmé, chez les apprenants confiants, l'effet bénéfique de l'autonomie : un examen du travail effectué et des propos avancés lors de l'entretien montre que leur motivation est restée élevée tout au long de la formation.

Il n'en va pas de même pour les moins confiants. Ce qui frappe lorsqu'on les interroge, c'est leur besoin - qu'ils reconnaissent volontiers - d'avoir quelqu'un qui les oblige à travailler. Pour eux, le lieu de contrôle doit être clairement externe. Si cette caractéristique semble en contradiction avec la théorie de l'auto-détermination, celle-ci est en partie résolue si l'on prend en compte d'autres caractéristiques associées à ce même profil d'apprenants.

D'abord leur niveau en anglais est plutôt faible, ce qui entraine des difficultés supplémentaires. La capacité cognitive de ces étudiants est en effet déjà largement accaparée par le contenu linguistique de la tâche, lui laissant peu de possibilités de s'en écarter, ne serait-ce que pour demander de l'aide (Raby, 2007). Ces apprenants ont peu sollicité les tuteurs car ils cherchaient des instructions précises comme celles que pourraient donner un enseignant. Comme ils manquent de connaissances métacognitives qui leur auraient permis de prendre du recul, ils se retrouvent enfermés dans une suite de tâches qui finissent par perdre toute cohérence. Leur motivation s'en trouve forcément affectée.

Par ailleurs, leur faible niveau linguistique, même s'il ne devrait pas être un problème dans un CAL, le devient lorsqu'il est accompagné d'une image négative de soi («Je suis nul en anglais, je le sais»). On est proche de l'incapacité apprise (Diener et Dweck, 1978), où l'apprenant, convaincu qu'il n'y arrivera pas, pense que ce n'est pas la peine d'essayer. Si ce profil extrême est plutôt rare, ce n'est pas non plus commun de se lancer volontairement dans une activité associée à de mauvais souvenirs.

Pour ce type d'apprenants, qui voient l'autonomie non pas comme un moyen d'améliorer leur niveau linguistique mais comme une difficulté supplémentaire à gérer, ce serait un non-sens de l'imposer ou l'exiger. Le CAL se doit de prendre en compte cette population tout en gardant l'autonomie comme un objectif affiché. Pour que ce type d'apprenant y adhère, au moins partiellement, avant la fin de sa formation, le personnel du CAL doit montrer qu'il cherche à le comprendre 
et à l'aider. Il importe d'abord de bien cerner son niveau linguistique afin de proposer des tâches à sa portée, si besoin dans un ordre défini. Se montrer compréhensif par rapport à son expérience passée, cause d'un manque éventuel de confiance, est également important : se sentir déprécié à cause d'un niveau jugé insuffisant, est fortement démotivant. Enfin, montrer que l'on comprend le désarroi que peut ressentir un apprenant face à l'autonomie permet de réduire son hostilité ou son angoisse. Le travail en groupe peut être rassurant à cet égard, et ce n'est pas inutile de penser et présenter le CAL comme un espace de travail collaboratif plutôt qu'une juxtaposition de ressources séparées. Signaler que l'autonomie est souhaitable, tout en admettant que chacun ne s'engage sur ce chemin que lorsqu'il se sent prêt, ôte la pression immédiate provoquée par la nécessité de gérer son parcours. La devise du CAL pourrait donc être : d'abord donner confiance et motiver, et l'autonomie viendra - peut-être. À ce moment-là on peut espérer que s'installe le cercle vertueux, où autonomie et motivation se renforcent mutuellement.

\section{RÉFÉRENCES BIBLIOGRAPHIQUES}

Albero B. (2002) : «L'autoformation en contexte institutionnel : entre la contingence et l'utopie», dans G. Le Meur (éd.), Université ouverte, formation virtuelle et apprentissage, Paris, L'Harmattan, p. 459-483.

BANDURA A. (2003) : Auto-efficacité: le sentiment d'efficacité personnelle, Bruxelles, De Boeck.

Benson P. (2001): Teaching and researching autonomy in language learning, Harlow, Pearson Education.

Clément R., Dörnyei Z. et Noels K. A. (1994) : «Motivation, selfconfidence and group cohesion in the foreign language classroom », Language Learning, vol. 44, p. 417-448.

Cotterall S. (1995) : «Readiness for autonomy: Investigating learner beliefs», System, vol. 23, n 2, p. 195-205.

Cotterall S. (1999) : «Key variables in language learning: What do learners believe about them?», System, vol. 27, p. 493-513.

DECI E. L. et RYAN R. M. (1985) : Intrinsic motivation and self-determination in human behaviour, New York, Plenum.

DeCI E. L. et Ryan R. M. (2002) : Handbook of self-determination research, Rochester, The University of Rochester Press.

Diener C. I. et Dweck C. S. (1978) : "An analysis of learned helplessness: Continuous changes in performance, strategy and achievement 
cognitions following failure», Journal of Personality and Social Psychology, vol. 36, p. 451-462.

Flavell J. H. (1979) : «Metacognition and cognitive monitoring: A new area of cognitive developmental enquiry », American Psychologist, vol. 34, p. 906-911.

Holec H. (2000) : «Le CRAPEL à travers les âges », Mélanges, $\mathrm{n}^{\circ} 25$, p. 5-12.

Mangenot F. et Nissen E. (2006) : «Collective activity and tutor involvement in e-learning environments for language teachers and learners », Calico Journal, vol. 23, no 3, p. 601-622.

Mozzon-McPherson M. (2007) : «Supporting independent learning environments: An analysis of structures and roles of language learning advisers », System, vol. 35, no 1, p. 66-92.

NARCy-Combes J.-P. (2003) : «Être tuteur, de la théorie à une pratique... », dans A. Cazade (éd.), ASp, n 41-42, «Pratiques et recherches en Centres de Langues », p. 21-34.

Pintrich R. (1999) : "The role of motivation in promoting and sustaining self-regulated learning », International Journal of Educational Research, 31, p. 459-470.

Prince P. (2008) : «La motivation dans un Centre de Langues, ou le pari de l'autonomie», Les Langues Modernes, $\mathrm{n}^{\circ}$ 3, p. 37-43.

RABY F. (2007) : «A triangular approach to motivation in Computer Assisted Autonomous Language Learning (CAALL)», ReCALL, vol. 19, n 2 , p. 181-201.

Raby F., Baillé J, Bressoux P. et Chapelle C. (2003) : «Ergonomic theory and practice: What language learners do in a self-access room», dans A. Cazade (éd.), ASp, n 41-42, «Pratiques et recherches en Centres de Langues », p. 67-84.

SinclaIR B. (1999) : «Wrestling with a jelly: The evaluation of learner autonomy », dans B. Morrison (éd.), Experiments and Evaluation in Self-Access Language Learning, Hong Kong, HASALD, p. 95-110.

Ushioda E. (1996) : Learner Autonomy 5: The role of Motivation, Dublin, Authentik.

Wenden A. (1998) : «Metacognitive knowledge and language learning», Applied Linguistics, vol. 19, no 4, p. 515-537. 


\section{ANNEXE 1}

Questionnaire dont la première partie traite de l'autonomie, la deuxième de la confiance.

Indiquez si vous êtes tout à fait d'accord $(+++)$, plutôt d'accord $(++)$, un peu d'accord (+), ou légèrement pas d'accord (-), plutôt pas d'accord (--), pas d'accord du tout (---) avec les affirmations suivantes :

\begin{tabular}{|c|c|c|c|c|c|c|c|}
\hline & J'ai besoin d'un professeur pour... & +++ & ++ & + & - & -- & --- \\
\hline 1 & expliquer le sens d'un texte & & & & & & \\
\hline 2 & évaluer mon travail & & & & & & \\
\hline 5 & expliquer des points de grammaire & & & & & & \\
\hline 7 & choisir le rythme de mon travail & & & & & & \\
\hline 10 & définir les objectifs & & & & & & \\
\hline 11 & me stimuler pour travailler & & & & & & \\
\hline 13 & corriger mes erreurs & & & & & & \\
\hline 15 & expliquer des références culturelles & & & & & & \\
\hline 16 & me donner le sens du vocabulaire utile & & & & & & \\
\hline 18 & me dire ce qu'il faut faire à chaque cours & & & & & & \\
\hline 19 & $\begin{array}{l}\text { Mon niveau en anglais est un handicap } \\
\text { pour moi }\end{array}$ & & & & & & \\
\hline 21 & $\begin{array}{l}\text { J'ai toujours eu de bonnes notes en } \\
\text { anglais à l'école }\end{array}$ & & & & & & \\
\hline 23 & $\begin{array}{l}\text { En ce qui concerne mes capacités en } \\
\text { anglais, j'ai confiance en moi }\end{array}$ & & & & & & \\
\hline 26 & $\begin{array}{l}\text { L'idée de parler anglais devant un groupe } \\
\text { me stresse }\end{array}$ & & & & & & \\
\hline 27 & $\begin{array}{l}\text { Aller au cours d'anglais ne m'apporte } \\
\text { rien parce que je n'arrive pas à suivre }\end{array}$ & & & & & & \\
\hline 29 & $\begin{array}{l}\text { Je n'ai pas une bonne oreille pour les } \\
\text { langues }\end{array}$ & & & & & & \\
\hline 31 & $\begin{array}{l}\text { Dans l'ensemble je garde de bons } \\
\text { souvenirs des classes d'anglais dans } \\
\text { le secondaire }\end{array}$ & & & & & & \\
\hline 33 & $\begin{array}{l}\text { Pour réussir en anglais il faut être doué(e) } \\
\text { pour les langues }\end{array}$ & & & & & & \\
\hline 34 & $\begin{array}{l}\text { J'aurais pu faire des études d'anglais } \\
\text { sans trop de difficultés si j'avais voulu }\end{array}$ & & & & & & \\
\hline 35 & $\begin{array}{l}\text { J'ai pris du retard en anglais et je dois le } \\
\text { rattraper }\end{array}$ & & & & & & \\
\hline
\end{tabular}




\section{ANNEXE 2}

Extrāits d'entreti ${ }^{i}$ ens āvec deũx ptũdiānts dont les scores moyens āũ âũest ${ }^{1}$ onnāire, sũr ũn māximũm de 5 , figũrent dāns le tābleãũ ci-dessoũs.

\begin{tabular}{|c|c|c|c|c|}
\cline { 2 - 5 } \multicolumn{1}{c|}{} & \multicolumn{2}{c|}{ début } & \multicolumn{2}{c|}{ fin } \\
\cline { 2 - 5 } \multicolumn{1}{c|}{} & confiance & autonomie & confiance & autonomie \\
\hline 1 & 2,5 & 1,7 & 2,9 & 2,8 \\
\hline 2 & 1,2 & 2,8 & 1,6 & 2,2 \\
\hline
\end{tabular}

\section{Sujet 1}

Pendant le collège et le lycée j'ai eu deux enseignants géniaux, sur 8 ans ça fait pas beaucoup, donc du coup j'ai dit que ça peut être une chance pour moi, parce qu'avec tout ce qu'il y a, entre les DVD, les cassettes, les cédéroms, tout ça, ça peut être une chance, c'est pour ça que je l'ai fait de bon cœur. Du fait qu'on se prenne en main, j'arrivais, je vais faire ce cédérom, je vais faire ci, je vais faire ça, et du coup j'étais complètement autonome, c'était plus la leçon de grammaire, le prof qui dit il faut apprendre les modaux comme ça. Je ne suis toujours pas bonne mais j'ai l'impression d'avoir fait un petit plus et ce petit plus je l'ai fait toute seule, avec l'aide du CALUP (Centre d'apprentissage des langues de l'Université de Provence), mais bon, c'était pas avec mes amis. Sauf pour le pairwork, bien sûr, et ça... le fait d'être à deux avec quelqu'un qu'on connait, on parlait plus facilement, surtout en France je trouve qu'on parle anglais avec un accent français, et que là, à deux on essayait plus d'être dedans que... ce qu'on oserait peut-être pas faire dans une classe. On est plus en confiance, moins... il n'y a plus le regard du prof ou des autres élèves, donc on est plus à l'aise à deux en pairwork. Mais sinon j'ai l'habitude de travailler seule, même dans les autres matières... C'est ça qui est bien, qu'on peut choisir, faire ceci ou cela, et je pense... bon, c'était pas toujours évident de savoir où j'allais, si je faisais des progrès. Mais je crois que oui. C'est peut-être juste une impression, mais je crois que si on essaie vraiment de progresser, on peut.

\section{Commentaire}

Cette étudiante partait d'un niveau de confiance moyen $(2,5)$ et surtout elle s'estimait très peu autonome $(1,7)$. Sa volonté de profiter autant que possible de l'autoformation s'est avérée payante, toutefois, dans la mesure où elle a su transférer à l'apprentissage de l'anglais une autonomie déjà acquise dans d'autres matières. Aidée en cela par l'absence d'un regard «contrôlant», elle est passée de la simple appréciation de la nouveauté du système et du choix de matériel pédagogique à un début de réflexion métalinguistique. Celle-ci n’est 
pas encore bien développée, mais l'étudiante s'est manifestement posée des questions sur ses progrès linguistiques et a trouvé des éléments qui lui permettent de croire qu'elle en fait. Ce résultat positif provient aussi de ses attentes réalistes au départ : elle sait en effet qu'un «petit plus », c'est déjà quelque chose de significatif. Ses réponses au questionnaire montrent qu'à la fin de la formation, elle fait preuve d'une autonomie plus développée pour tout ce qui concerne la recherche d'explications, mais elle ressent davantage le besoin d'un professeur qui la stimule pour travailler. Sa préférence pour le travail solitaire l'empêche-t-elle de chercher la stimulation qu'aurait pu fournir un travail en groupe ? En tout cas, ce n'est pas irréaliste de supposer que si elle continue à trouver de l'aide pour la soutenir dans ses efforts, elle arrivera à une forme de motivation intrinsèque plus affirmée, voire à une pleine autonomie.

\section{Sujet 2}

Toute seule je n'y arrive pas, j'ai tellement de lacunes que je ne m'en sors pas toute seule, donc j'ai besoin de quelqu'un à côté de moi qui me guide, et dès que je fais une erreur me corrige de suite parce que sinon l'erreur, je la garde jusqu'à la fin. J'ai un très mauvais niveau d'anglais, donc du coup j'ai pas beaucoup d'autonomie et c'est vrai que toute seule en fait, je voulais, je pensais pouvoir et en fait non, je n'y arrive pas... Je sais pas, je sais pas ce qu'il faut, je ne comprends pas, c'est pour ça. Si jamais c'est tout en anglais dès le départ je ne comprends pas, donc il faut qu'on m'aide, il faut qu'on m'aiguille, il faut surtout qu'on traduise des fois, il faut quelqu'un pour faire ça. Toute seule, je peux pas... La tutrice m'a dit qu'il fallait que je continue à travailler parce que j'ai commencé à revoir les bases, à reprendre des bouquins de premier niveau avec le présent, le verbe être, et donc elle m'a dit que je devais continuer, me fixer de petits objectifs comme ça, mais bon... il faut que je parte là-bas et ça ira beaucoup mieux après. Le système qu'on nous fait au lycée, au collège, je n'arrive pas... Travailler ici c'est très différent de ce que j'ai connu au lycée, les tuteurs comprennent qu'on puisse avoir des difficultés, des lacunes, qu'on puisse être nulle, donc ils sont ouverts à ça, alors qu'avant les professeurs que j'avais, j'étais nulle dans mon coin, on s'occupait pas de moi... L'anglais est devenu plus agréable, c'est vrai, j'ai plus apprécié cette année l'anglais qu'avant. Mais bon, je suis toujours aussi nulle, il faut que je parte un an. Si je n'étais pas obligée je n'en ferais pas, mais là je suis obligée. C'est ma bête noire. Mais je ne vais pas me rendre malade pour ça, c'est quelque chose de normal, enfin normal... je suis nulle, je l'assume, je le sais et voilà, je fais avec.

Quand vous dites ça, c'est comme si vous l'acceptez.

Non... Oui, je l'accepte. J'essaye de travailler pour être... Mais je peux pas non plus devenir bilingue en deux deux, donc je vais pas me rendre malade. Si jamais j'ai l'opportunité de faire des, de m'améliorer, bien j'améliore, après bon ben... C'est pas une de mes choses primordiales que je dois faire cette 
année, c'est pas l'anglais... Ici, c'est bien, tout le choix qu'on a, mais c'est presque trop de choix. Quelqu'un comme moi, il me faut quelqu'un à côté tout le temps.

\section{Commentaire}

Si le niveau de confiance de cette étudiante a augmenté comme celui de la précédente, son degré estimé d'autonomie a baissé. Si l'étudiante précédente a sans doute sous-estimé sa capacité à l'autonomie, celle-ci l'a surestimée. Animée par une même volonté de profiter d'un nouveau système d'apprentissage, que par ailleurs elle apprécie, elle s'est heurtée à une incapacité à surmonter ses difficultés linguistiques. Elle est dans un cercle vicieux proche de l'incapacité apprise, où par manque de confiance elle renonce à chercher une solution par elle-même. De ce fait, malgré des souvenirs négatifs de l'école, elle réclame une présence constante, mais qui serait cette fois bienveillante. Consciente de la difficulté à obtenir une aide personnalisée permanente, elle se réfugie dans l'espoir de partir en pays anglophone. Stratagème pour réduire son angoisse ou détermination réelle? Difficile à dire, mais son désarroi est frappant, et de toute évidence elle a dû ériger des défenses contre la perception qu'elle a de sa propre «nullité». Pour le personnel du CAL, c'est un cas difficile, douloureux même, qui souligne la nécessité d'une écoute et d'un soutien précoces, mais qui montre aussi, sans doute, les limites du dispositif d'apprentissage quel qu'il soit. 